\title{
Iatrogenic esophageal and tracheal perforation with tracheoesophageal fistula following emergency intubation in a patient with cervical esophageal cancer
}

\author{
Akram Alkrekshi ${ }^{1}$ and Hazim Bukamur ${ }^{2}$ \\ ${ }^{1}$ MetroHealth Medical Center \\ ${ }^{2}$ Texas Tech University System
}

June 24, 2020

\begin{abstract}
A 52-year-old female with a recently diagnosed esophageal cancer presented with hematemesis that needed emergent endotracheal intubation (ETT). Two-days later following respiratory compromised, computed tomography imaging showed an abnormally placed ETT which traversed from esophagus to trachea resulting in tracheoesophageal fistula. There was no pneumomediastinum or pneumothorax.
\end{abstract}

Iatrogenic esophageal and tracheal perforation with tracheoesophageal fistula following emergency intubation in a patient with cervical esophageal cancer

Akram Alkrekshi ${ }^{1}$, Hazim Bukamur ${ }^{2}$

1. Department of Internal Medicine, The MetroHealth System campus of Case Western Reserve University, Cleveland, OH, 44109, USA.

2. Department of Pulmonary and Critical Care Medicine, Texas Tech University Health Science Centre, Lubbock, TX, 79409, USA.

Corresponding Author: Akram Alkrekshi MD

Email : (aalkrekshi@metrohealth.org )

Address : Department of Internal Medicine 2500 Metrohealth Dr, Metrohealth Medical Center Cleveland, OH, 44109, USA

Authors contribution statement: Both authors have contributed significantly in the production of the manuscript.

Keywords : iatrogenic, tracheoesophageal fistula, esophageal cancer

Key Clinical Message:

Iatrogenic tracheoesophageal fistula through direct penetration of esophageal and tracheal walls is exceedingly rare. Body tissues sealing around the tube may result in a delayed diagnosis. Pneumomediastinum and pneumothorax may be absent.

Manuscript:

A 52-year-old female with a history of tongue squamous carcinoma treated with surgery and radiotherapy 6 -years prior. Recent work-up for dysphagia revealed an ulcerated friable mass at the post-cricoid region that 
extended $8.5 \mathrm{~cm}$ distally. Flexible bronchoscopy showed posterior mid-tracheal wall asymmetry. Biopsies confirmed cervical esophageal squamous carcinoma (T2N2M0). The patient presented with hematemesis complicated by asphyxiation and cardiopulmonary arrest. She was resuscitated with return of circulation within 4-minutes. Intubation was difficult; bougie and video laryngoscopy were used to place a size-6 endotracheal tube (ETT). Two days later, the patient developed acute respiratory failure. Computed tomography imaging showed bilateral consolidation and abnormally placed ETT which traversed from esophagus to trachea resulting in tracheoesophageal fistula (TEF). There was pneumomediastinum, or pneumothorax (figure 1-4). Antibiotics started, and enteral-feedings held. Three days later, gastrostomy-tube placement, tracheostomy, and ETT removal in the operating theater were done. Radiotherapy and chemotherapy followed.

TEF is rarely iatrogenic, and usually due to posterior tracheal wall perforation during intubation or posterior wall erosion from pressure by an overinflated endotracheal cuff $^{1}$. This case is unusual as TEF was due to an errantly placed ETT. Maintaining the airway through TEF until tracheostomy and supportive measures resulted in a satisfactory outcome.

1. Mooty RC, Rath P, Self M, Dunn E, Mangram A. Review of Tracheo-Esophageal Fistula Associated with Endotracheal Intubation.J Surg Educ . 2007;64(4):237-240. doi:10.1016/j.jsurg.2007.05.004

List of figures legend

1. Figure 1: Endotracheal tube in the esophagus

2. Figure 2: Endotracheal tube traversing the esophagus

3. Figure 3: Endotracheal tube in the trachea

4. Figure 4: Endotracheal tube traversing the esophagus into the trachea

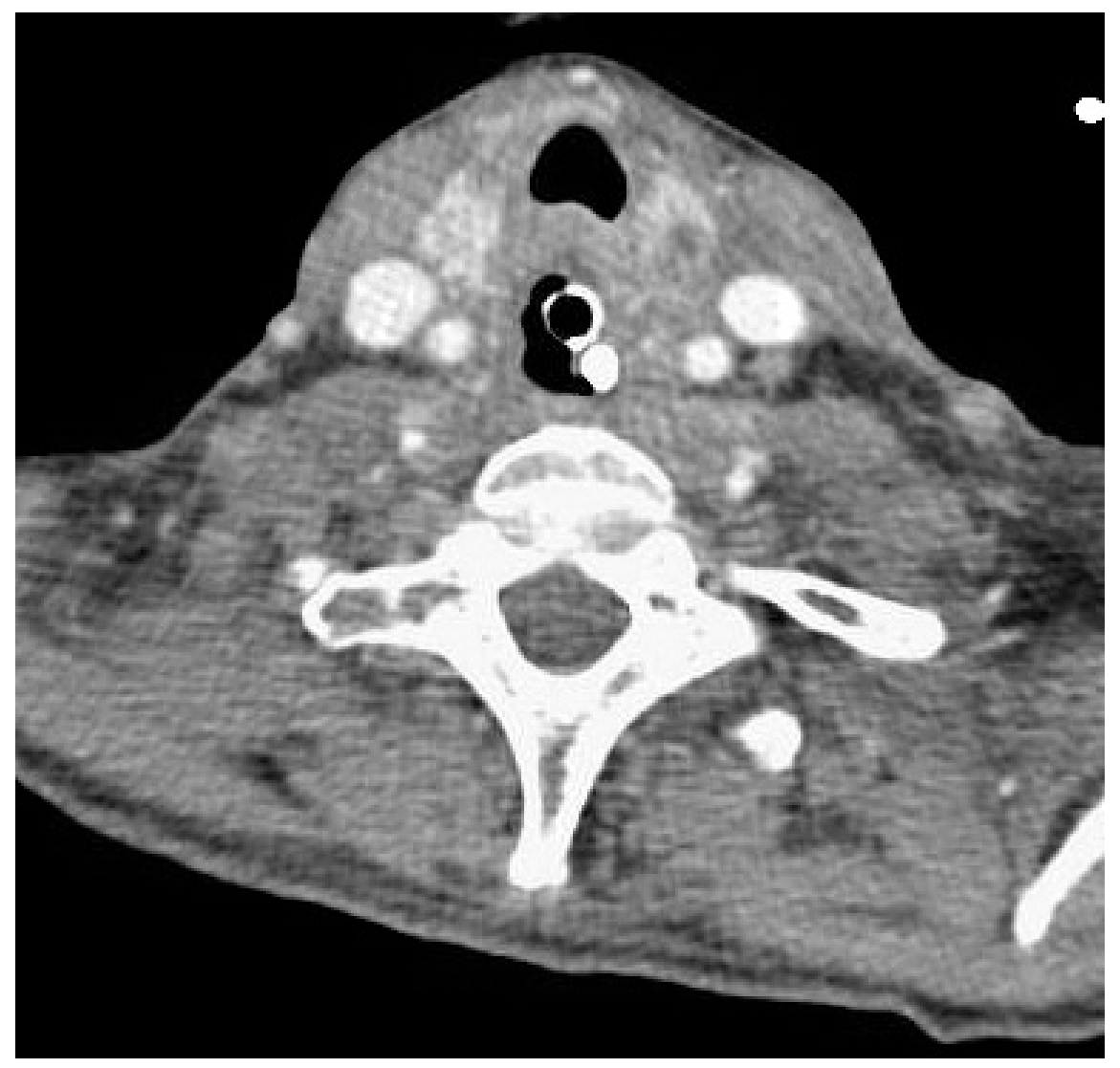



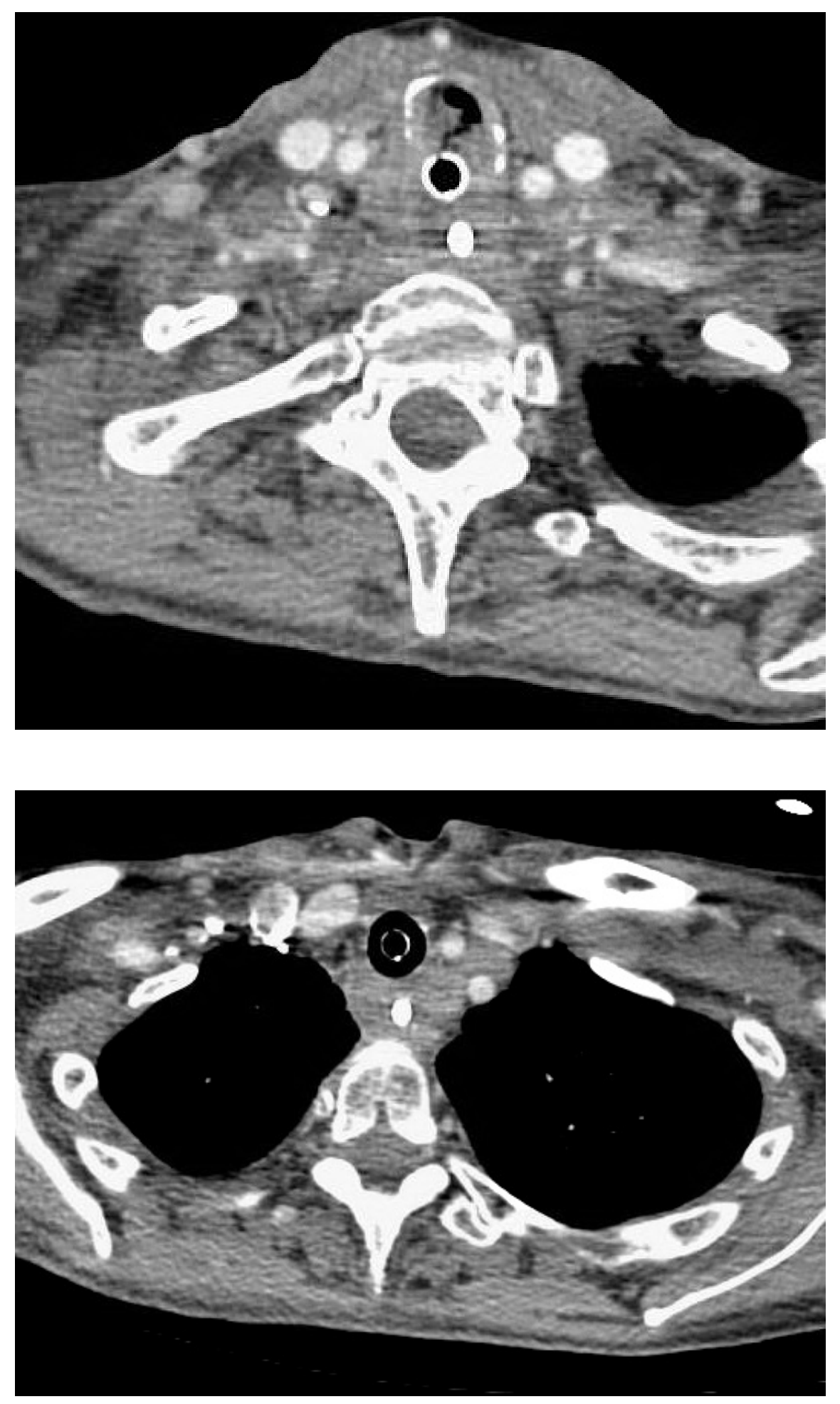


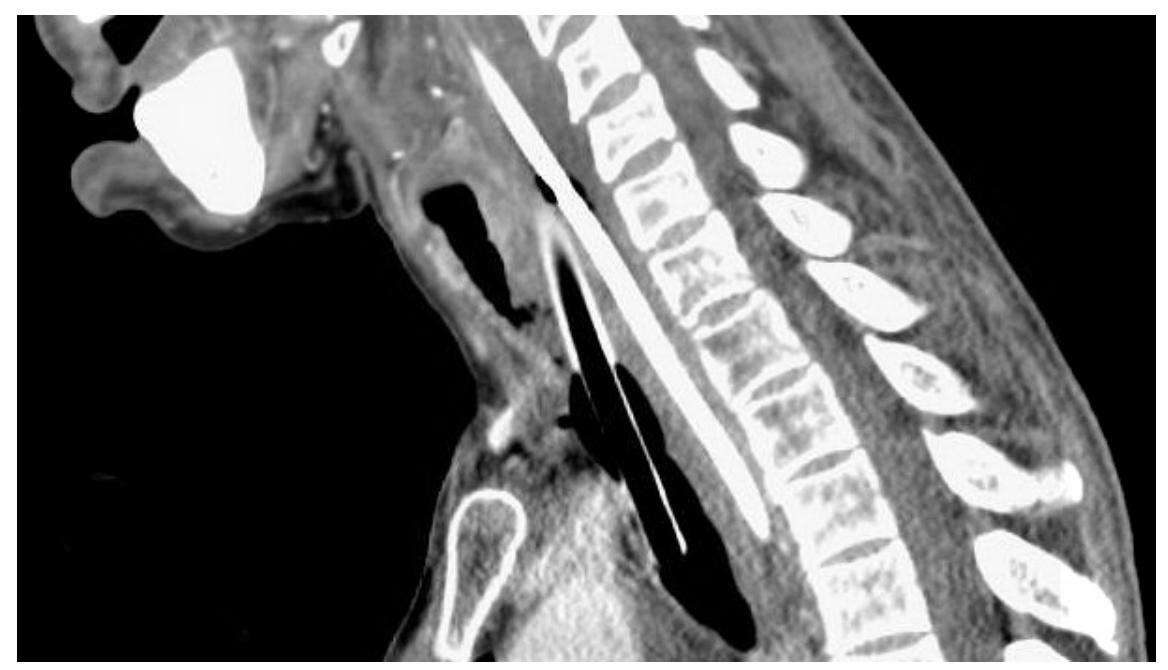

